\title{
An Actuarial Model of Autonomous Vehicles
}

\author{
Heyan Wang*, Jun Xie and Chi Mo \\ Department of Mathematics and Information Technologies, Guangxi University, Guang Xi, China \\ ${ }^{*}$ Corresponding author
}

\begin{abstract}
The technology of autonomous vehicles is growing fast in current time. On one hand, the popularization of autonomous vehicles is expected to greatly reduce the occurrence of traffic accidents, meanwhile lowers the income of premium for insurance companies; on the other hand, the decrease of traffic accidents also reduces the amount of claim for these companies. Based on the actuarial model of manual vehicles and the data of current autonomous vehicles, this paper proposes an actuarial model for autonomous vehicles, and then computes the corresponding premium. Through comparison between the premiums of autonomous vehicles and manual vehicles, we find that autonomous vehicles reduce not only the financial burden on their owners, but also the burden and cost of health on the entire society.
\end{abstract}

Keywords-autonomous vehicles; actuarial model; manual vehicles; poisson distribution

\section{INTRODUCTION}

Bridget Carlin once said, "cars are among the biggest mobile devices"[1]. As the development of autonomous vehicle technologies, traditional insurance industry is faced with huge challenges. Major tech companies, such as Google, Baidu, have invested a large amount of money into the research and development of autonomous vehicles; and it is expected that autonomous vehicles will gradually enter the market till 2021 ${ }^{[1]}$ World Economic Forum (WEF) has estimated that the digitalization of car industry will create a value of 67 billion dollars and result in a social benefit of 3.1 trillion dollars, including the improvement of autonomous vehicles, the interconnection of car users, and the perfection of the entire transportation industry ${ }^{[1]}$. Based on above facts, it is believed that the proportion of insurance business for autonomous vehicles will increase in the coming years. On the other hand, to the authors' knowledge, there is still no research and analysis concerning the actuarial model of autonomous vehicles. To fill the research gap, this study proposes an actuarial model of autonomous vehicles, and then compares the proposed model to that of manual vehicles in order to draw some managerial insights.

The remainder of this paper is organized as follows: Section 2 introduces the actuarial model for manual vehicles; Section 3 then introduces the proposed actuarial model for autonomous vehicles based on above model. Through a case study, we illustrate that autonomous vehicles will greatly reduce the financial burden of their owners in Section 4. Section 5 then concludes the paper.

\section{ACTUARIAL MODEL FOR MANUAL VeHICLES ${ }^{[2]}$}

\section{A. Model Parameters ${ }^{[3]}$}

We first briefly introduce the parameters in the actuarial model for manual vehicles. The parameters include: travel distance per year; type of vehicle; location of parking; rate of accidents; the age of driver; the occupation of driver. We note that these factors will be considered as exogenous parameters in the model.

\section{B. Accident Rate Model ${ }^{[4]}$}

In conventional actuarial model, the accident of vehicles is modeled as a Poisson distribution. For convenience of readers, we briefly introduce Poisson distribution in this section.

Assume $X$ is the random variable representing the number of accidents happened within a given period. By the definition of Poisson distribution, the probability that accidents occurs $k$ times is given by:

$$
P(x=k)=\frac{\lambda^{k}}{k !} e^{-\lambda}, k=1,2, \ldots
$$

Where $\lambda$ is the parameter of this Poisson distribution. Figure I shows a Poisson distribution with $A=3.5$.

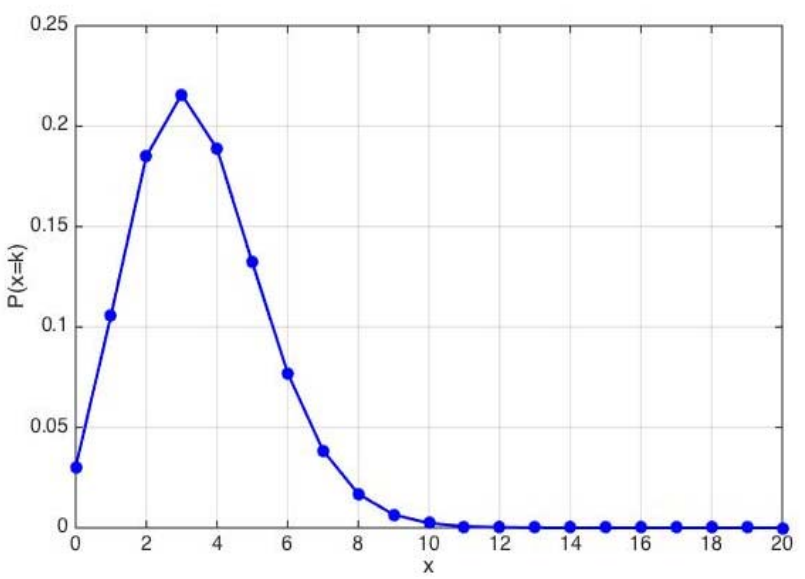

FIGURE I. ILLUSTRATION OF POISSON DISTRIBUTION WITH

$$
\lambda=3.5
$$

Poisson distribution has the following properties:

a) If a set of insurances each obeys independent Poisson distributions, then the sum of the insurance obeys 
Poisson distribution with the parameter equals to the sum of parameters of these independent distributions.

b) The expectation and variance of a Poisson distribution are equal in value.

c) The occurrence of accidents obeying Poisson distribution is invariant to the start time; and the probability that more than two accidents occurs within an extremely short period can be viewed as zero.

\section{Claim Amount Model}

In the theory of actuarial model for manual vehicles, there are various assumption of distribution model including exponential distribution, Gamma distribution, Pareto distribution, etc. ${ }^{[3]}$ In this paper we employ the most popular exponential distribution. The probabilistic distribution function (PDF) of exponential distribution is given by:

$$
f(x)= \begin{cases}\theta e^{-8 x}, & x \geq 0 \\ 0, & x<0\end{cases}
$$

Where $\theta$ is the parameter of the distribution. Figure II shows an exponential distribution with $\theta=1$.

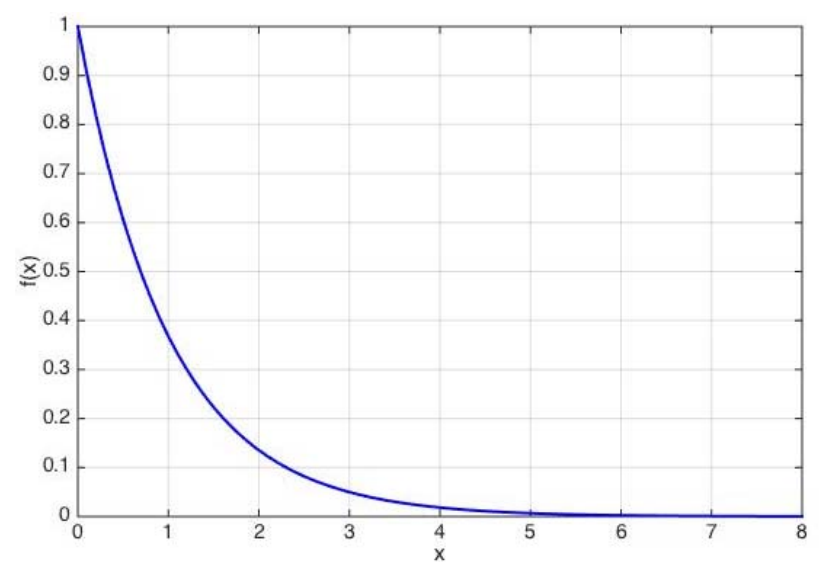

FIGURE II. ILLUSTRATION OF EXPONENTIAL DISTRIBUTION WITH

$$
\theta=1
$$

The most important property of an exponential distribution is the so-called "memoryless property", which indicates that $P^{\prime}(x \geqslant t+s \mid x \geqslant t)=P(x \geqslant g)$.

\section{Premium Pricing Model for Manual Vehicles}

According to $\mathrm{Yu}$ (2010), for premium pricing of manual vehicles, we assume:

a) The accident rate obeys Poisson distribution, and the claim amount obeys exponential distribution;

b) The occurrence of accidents of different vehicles is independent.

c) (Fairness) The premium must not exceed the maximum claim amount; d) (Profitability) The premium must be larger than or equal to the average of claim.

With above assumptions, the premium pricing can be given by the following equation:

$$
P=E(S)+\beta \sqrt{D(S)}
$$

Where $E(S)$ and $D(S)$ represents the expected claim amount and variance, respectively. When $S$ is large enough, setting $\beta=3$ can guarantee a rate of profitability to $99.7 \%$, which can greatly reduce the risk.

In practice, the gross premium is designed to be the sum of Eq.(3) and an additive part. Then the gross premium is computed by:

$$
W_{1}=c_{1} m \times\left(1+a_{1} \sqrt{c_{1}}+a_{2}\right)
$$

Where $W_{1}$ represents the gross premium of manual vehicle, $c_{1}$ is the accident rate of manual vehicles, $m$ represents the maximum amount of claim, $a_{1}$ and $a_{2}$ are two parameters to guarantee profitability.

\section{ACtUARIAl MODEl FOR AutONOMOUS Vehicles}

The actuarial model of autonomous vehicles can be obtained with similar procedure shown above. However, with the technological development of autonomous vehicles, it is expected that the accident rate can be greatly reduced. Assuming the new accident rate is $c_{2}$, then the gross premium for autonomous vehicle is:

$$
W_{2}=c_{2} m \times\left(1+a_{1} \sqrt{c_{2}}+a_{2}\right)
$$

Where $W_{2}$ represents the gross premium of autonomous vehicles.

\section{CASE STUDY}

In this section, we use a case study to show the reduction of financial burden for car owners with the introduction of autonomous vehicles. Assuming an insurance company is trying to pricing the premium for car accident with a maximum claim of 20,000 dollars in 2017. The historical data shows that for 5000 vehicles, the amount of claim is 2.5 million dollars (500 dollars per vehicle) in 2014, and it is 4 million (800 dollars per vehicle) in 2015, 3.8 million (760 dollars per vehicle) in 2016. The parameters $a_{1}$ and $a_{2}$ are set to be 3 and 0.3 .

Since we assume the accident rate obeys Poisson distribution, then the accident rate per vehicle per year can be computed by:

$$
c_{1}=\frac{(2.5+4+3.8) \times 10^{6}}{5000 \times 2 \times 3}=0.0343
$$


Then we can compute the gross premium to be:

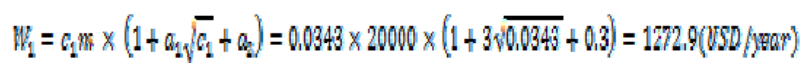

Then we consider the gross premium for autonomous vehicles. To model the improved safety of autonomous vehicles compared to manual vehicles, we let $c_{2}=\alpha c_{1}$, where $0 \leqslant \alpha \leqslant 1$ is a factor of accident reduction; the smaller $a$ is, the safer of autonomous vehicles compared to manual ones. Figure III shows the value of $\frac{w_{2}}{w_{1}}$ as a function of $\alpha$, i.e., given a specific value of $a$, how much is the proportion of gross premium for autonomous vehicles compared to that of manual ones.

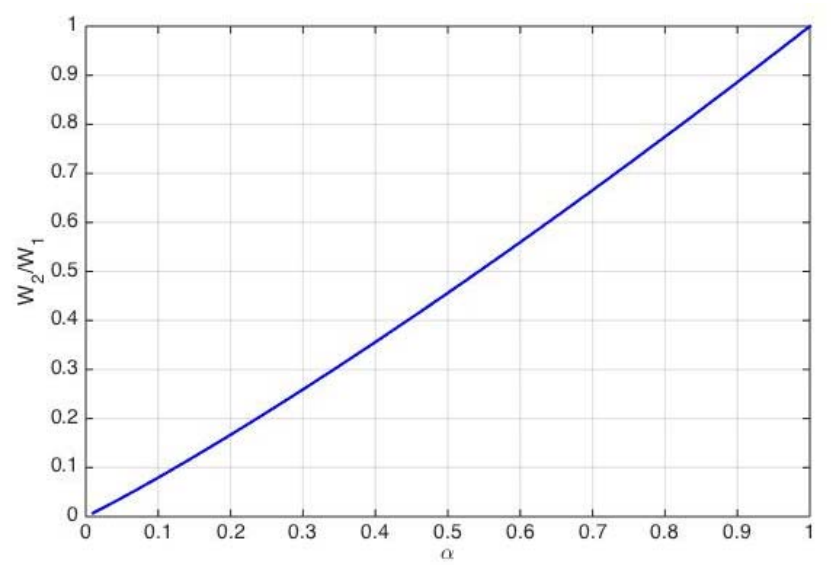

FIGURE III. REDUCTION OF PREMIUM W.R.T. REDUCTION OF ACCIDENT RATE

By Figure III, it is obvious that the premium pricing reduces with the accident rate; for example, when $\alpha=0.2$, i.e., the accident rate reduces to $20 \%$ of that of manual vehicles, each owner is required to pay only about $1272.9 \times 0.17=216.4(U S D$ ( year $)$. This will greatly reduce the financial burden on car owners.

\section{CONCLUSION}

In this paper, we propose an actuarial model for autonomous vehicles assuming the accident rate obeys Poisson distribution and the claim amount obeys exponential distribution; and through comparison with that of manual vehicles, we find that autonomous vehicles are expected to greatly reduce the pricing of premium for single car owner. This suggests that the development of autonomous vehicle technologies will not only benefit the life and health of people, but also relieve the financial burden on car owners to achieve economic efficiency.

\section{REFERENCES}

[1] West, D. M. Moving forward: Self-driving vehicles in China, Europe, Japan, Korea, and the United States.( 2016)

[2] Yu, J. A review of actuarial models for vehicles. Journal of Shanghai Financial School, 1(1), 14-19. (in Chinese) (2010)
[3] Zhang H.The factors that should be taken into consideration in the formulation of the third party liability insurance rate [J].Insurance research, 06,15:26-28. (in Chinese) (1994)

[4] Meng S., Yuan W. Actuarial model of automobile insurance and its application [J]. mathematical statistics and management, 20 (3): 61-64. (in Chinese) (2001) 